\title{
Estudiantes de primera generación en Chile: una aproximación cualitativa a la experiencia universitaria
}

\section{First-generation students in Chile: a qualitative approach to university experience}

\author{
Valentina Javiera SOTO HERNÁNDEZ \\ Universidad de Concepción (Chile)
}

Recibido: Diciembre 2014

Evaluado: Abril 2015

Aceptado: Junio 2015

\section{Resumen}

El propósito de este artículo es analizar la experiencia universitaria de un grupo de estudiantes de primera generación de tres universidades ubicadas en Concepción, Chile. En un contexto de fuerte masificación de la educación superior, la experiencia de los estudiantes cuyos padres completaron como máximo la educación secundaria, se ha transformado en un ámbito de incipiente interés. En función de los objetivos, la investigación fue abordada desde el paradigma constructivista y diseñada de acuerdo al método biográfico, cuya principal técnica de recolección de datos fueron las entrevistas semi-estructuradas. Los resultados de esta investigación, de carácter exploratorio, revelan que la construcción de un proyecto de estudios universitarios se encuentra notablemente influenciado por los padres, quienes se transforman en una fuente fundamental de apoyo. Asimismo, los estudiantes configuran tres significaciones a su experiencia universitaria: la movilidad social ascendente, la vocación y la retribución a los padres. Finalmente, se discuten los resultados a partir de los cuales se proponen nuevos interrogantes.

Palabras clave: estudiante universitario, proyecto, integración, movilidad social.

\begin{abstract}
The aim of this article is to analyse the university experience of a group of first- generation undergraduate students from three universities of Concepción, Chile. In a context of a strong massification of higher education, the experience of students whose parents completed secondary education at most has turned into a field of emerging interest. Depending on the objectives, the following research was approached from a constructivist paradigm and designed according to the biographical method, whose principal data collecting technique was the use of semi-structured interviews. The results of this exploratory study show that the building up of a university education project is greatly influenced by parents, who turn into an fundamental source of support. In the same way, students configure three relevant meanings to their
\end{abstract}


university experience: upward social mobility, vocation and gratitude to parents. Finally, the results are discussed and new questions are proposed.

Keywords: university student, project, integration, social mobility.

Las transformaciones de los sistemas educación superior se han producido en el contexto de las políticas públicas que favorecen su expansión. Este proceso ha implicado una transformación del perfil del estudiantado, gracias a la incorporación de estudiantes de sectores populares a un espacio universitario antes reservado a las clases altas. En este escenario, emergió la noción de estudiantes de primera generación, desde el cual se desarrolló esta investigación. Esta noción busca dar cuenta del efecto de la escolaridad de los padres en sus hijos a partir de una dicotomía: padres con educación superior y padres que no alcanzaron este nivel.

El sistema de educación superior chileno experimentó significativas transformaciones a causa de la implementación de reformas neoliberales impulsadas por la dictadura militar durante los años 80. Una de sus consecuencias fue el crecimiento explosivo de la matrícula de la educación superior, especialmente la universitaria (Rolando etal., 2010). Progresivamente, se produjo un aumento de las expectativas de los estudiantes y sus familias para acceder a este nivel educativo (Centro de Investigación y Desarrollo de la Educación [CIDE], 2012).

De esta forma, muchos estudiantes hijos de padres sin educación superior pudieron acceder a la universidad. No obstante, su experiencia es distinta a aquella de los hijos de universitarios: los estudiantes de primera generación presentan menores niveles de acceso y de obtener un título profesional. En este contexto, el objetivo de la investigación fue analizar la experiencia universitaria en un grupo de estudiantes de primera generación, en la ciudad de Concepción, Chile. En particular, se buscó comprender el proceso de articulación de las lógicas de acción de la experiencia y las posibles dificultades encontradas por estos estudiantes.

El artículo está estructurado como sigue. Primero, a modo de marco referencial, serán presentados el concepto de experiencia universitaria y la noción de estudiantes de primera generación (en adelante, EPG), a partir de diversas investigaciones. Enseguida, se detallará la metodología y procedimientos utilizados, para luego analizar los principales resultados, los que serán examinados y discutidos, de manera de proponer nuevas interrogantes.

\section{La experiencia universitaria}

El concepto de experiencia, desde una perspectiva sociológica, fue planteado por François Dubet. A partir de una investigación en instituciones escolares francesas, Dubet y Martucceli (1996) propusieron el concepto de "experiencia escolar". La experiencia se construye en tanto que "prueba", en la cual los estudiantes tienen el imperativo de aprender a combinar las lógicas de acción de integración, estrategia y subjetivación. De tal modo, la trayectoria escolar se constituye como un proceso de formación de la individualidad, cuyo resultado es la capacidad de combinar estas 
lógicas (Dubet y Martuccelli, 1996, p. 330). El concepto de experiencia universitaria, gestado a partir de una investigación en universidades francesas, tiene el mismo sentido que el de experiencia escolar.

En el contexto de la masificación del acceso a la universidad, Dubet (1994b) considera que las variables clásicas de la sociología no permiten dar cuenta de la experiencia de los estudiantes. Por esto, propone analizar la experiencia universitaria a partir de tres dimensiones: proyecto, integración y vocación, que en términos de experiencia escolar corresponden a las lógicas de acción de estrategia, integración y subjetivación, respectivamente (Dubet, 1994a).

(1) El proyecto es "la representación subjetiva de la utilidad de los estudios, por un actor capaz de definir los objetivos, de evaluar las estrategias y sus costos" (Dubet, 1994a, p. 513). Tres tipos de proyectos son propuestos: (a) proyecto profesional, en el cual la prioridad es obtener el diploma universitario como medio de acceso al mercado laboral; (b) proyecto escolar, donde los estudiantes utilizan la misma lógica que en secundaria, es decir, acumular capital escolar, más frecuente durante los primeros años de universidad; y (c) ausencia de proyecto, que implica la falta de expectativas respecto a los estudios superiores, lo cual puede conducir a la deserción (Dubet, 1994b).

(2) La integración se refiere a la socialización de los estudiantes en la institución y en el medio estudiantil, que no sólo es académica, puesto que la sociabilidad entre pares cumple un rol significativo.

(3) Para Dubet (1994b), el concepto de vocación se orienta hacia una significación "ética" de los estudios. Desde esta dimensión, la experiencia universitaria es vivida como un "sentimiento de realización intelectual" (p. 517) gracias a los nuevos conocimientos. "La vocación es una de las dimensiones centrales de esta experiencia, aquella que permite sentirse "verdaderamente estudiante", en la medida en que ella define la influencia (...) de la formación sobre la personalidad, sobre las formas de ver el mundo y de situarse en él" (Dubet, 1994b, p. 519).

A partir de estas tres dimensiones, Dubet (1994a) propone "figuras de experiencia universitaria". La figura de los "verdaderos estudiantes" presenta las tres dimensiones en su grado "fuerte". Por el contrario, los estudiantes que viven el anonimato, sin un proyecto ni vocación son representados bajo la figura "malheur étudiant ${ }^{2}$ " y experimentan las tres dimensiones en su grado "bajo". Según Dubet (1994a), las figuras de "verdaderos estudiantes" y "malheur étudiant" se ubican en los polos extremos de las experiencias, entre los cuales se encuentra una gran variedad de figuras de estudiantes, puesto que la experiencia universitaria es diversa.

\footnotetext{
${ }^{1}$ Las citas de la bibliografía en francés fueron traducidas al español por la autora.

2 "Desgracia estudiantil", en traducción libre.
} 


\section{La transformación del perfil de los estudiantes universitarios}

La masificación de la educación universitaria ha implicado una transformación de perfil de los estudiantes. Previo a la masificación la universidad conformaba un espacio casi exclusivo para jóvenes provenientes de medios sociales favorecidos. Los estudiantes universitarios eran una élite. La figura del heredero de Bourdieu y Passeron (1964) reúne estas ideas. Los estudiantes de clases altas deben a su origen un conjunto de costumbres y actitudes apreciadas en el ámbito escolar. "Lo esencial de la herencia cultural se transmite de manera más discreta y más indirecta, e incluso en ausencia de todo esfuerzo metódico y de toda acción manifiesta" (Bourdieu y Passeron, 1964, p. 34). Los autores plantean que los conocimientos transmitidos en la escuela están asociados a valores sociales cercanos a aquellos de la clase alta. Mientras que para los estudiantes de clases populares, el aprendizaje de estos valores es un proceso arduo.

La transformación del perfil de los estudiantes implica que "[...] la figura del estudiante no es más entonces la del heredero en búsqueda de distinción y de una "objetivación" de su capital cultural en un diploma" (Felouzis, 2001, p. 232). En la universidad masificada, la figura del heredero se diluye frente al advenimiento de los "nuevos estudiantes". De acuerdo a Felouzis (2008), los estudiantes de origen popular, desprovistos de conocimientos de la cultura universitaria, se enfrentan a un mundo ajeno; la universidad está simbólicamente alejada de ellos, ya que no poseen el capital cultural para comprender fácilmente las normas y los códigos de la comunicación universitaria, que a menudo son implícitos.

\section{Los estudiantes de primera generación}

La expresión de estudiantes de primera generación fue concebida en el marco de la expansión de la educación superior y el aumento de estudiantes procedentes de sectores populares. De acuerdo a Auclair etal. (2008), la definición más generalizada de EPG, utilizada en este trabajo, se refiere a hijos de padres sin diploma de estudios superiores $\mathrm{y}$ sin experiencia en este nivel educacional.

Chen y Carroll (2005) señalan que los EPG tienen más probabilidades de repetir y abandonar asignaturas; mientras que Groleau etal. (2010) concluyeron que el estatus de EPG determina el acceso y permanencia en la educación superior. Los resultados de investigaciones cualitativas dan cuenta de la complejidad de los procesos que experimentan estos estudiantes. De igual forma De acuerdo a Erlich y Boyer (2000) los EGP presentan sentimientos de exclusión y prácticas auto-excluyentes, lo que unido a su escasa familiaridad con la pedagogía universitaria provoca desajustes respecto a las exigencias académicas (Boyer et al., 2001). Reay et al. (2010) plantean, a partir de una investigación con estudiantes de clase trabajadora en universidades de Reino Unido, que ellos no sólo enfrentan desafíos académicos, sino también un trabajo sobre su propia identidad debido a las tensiones entre su origen social y el entorno universitario, especialmente en universidades de élite. A pesar de los similares orígenes sociales, Lehmann (2012) encontró trayectorias universitarias disimiles: mientras para algunos el paso por la universidad implicó realización personal; para otros, conllevó alienación 
y temor. De esta forma, la universidad conlleva "efectos contrastados y ambivalentes" para los "hijos de la democratización" (Beaud, 2002).

\section{Los padres frente a la escolaridad de sus hijos}

En entornos desfavorecidos, los padres a menudo son señalados como los responsables de las dificultades escolares de sus hijos, a causa, entre otras, de la falta de compromiso con su educación. En este sentido, Lahire (1995), estudiando familias de origen popular, desmiente lo que él denomina el "mito de los padres dimitidos". En efecto, aunque la familia posea un bajo capital cultural, la -educación puede tomar un "lugar simbólico" en la relación padres-hijos-escuela, contribuyendo al éxito escolar.

Los padres esperan que, gracias a la educación, sus hijos consigan un mejor empleo: "menos fatigante, menos sucio, menos mal pagado y más valorizado que el suyo" (Lahire, 1995, p. 398). Charlot (1999) plantea que el oficio de los padres funciona como "una referencia (positiva o negativa) en la construcción del sujeto" (p. 259), pero que en sí mismo no es relevante, sino el nivel de vida que conlleva. Esto es clave para los estudiantes ya que su aspiración es tener una vida diferente a la de sus padres.

La fuerte asociación del nivel socio-económico entre padres e hijos se manifiesta en la compleja ruptura de la transmisión inter-generacional de la pobreza. Investigando este tema en Israel, Gofen (2009) obtuvo resultados que sugieren que la familia tiene un rol clave para los EPG, constituyéndose más bien en una fuente de recursos que de restricciones. De acuerdo a su análisis, el mecanismo para romper la transmisión de la pobreza se desarrolla en tres aspectos. Primero, los padres otorgan un papel fundamental a la educación de los hijos, inscrita en un "horizonte temporal" de larga duración. Enseguida, las "relaciones interpersonales" referida a la disposición de los padres a realizar "sacrificios" por sus hijos para favorecer el acceso a la universidad. Finalmente, "valores de la familia", donde destacan la solidaridad y el respeto a los padres. Estos tres elementos confluyen en la implicación de los padres para promover los estudios superiores de sus hijos.

La implicación de los padres podría conllevar una disminución del "shock cultural" en la entrada a la enseñanza superior, según lo plantean McCarron y Inkelas (2006), citados en Gofen (2009). Galland y Oberti (1996) enfatizan la existencia de una relación entre la integración a la universidad y familia, manifestando que "los estudiantes mejor socializados en la universidad son probablemente aquellos cuyo proyecto escolar prolonga o desarrolla un proyecto familiar de integración o ascenso social por los estudios" (pp. 50-51). De tal forma, la existencia de un proyecto familiar de movilidad ascendente por medio de los estudios parece asociarse positivamente a la integración en la universidad. 


\section{Estudiantes de primera generación en Chile}

A pesar de la masificación de la educación superior, las investigaciones revelan persistentes desigualdades (Espinoza etal., 2009). En efecto, el acceso a la educación superior está determinado por el estatus socio-económico del estudiante: la representación del quintil de ingreso más alto es casi tres veces superior que el quintil más bajo (Brunner, 2011); además, el nivel de ingresos determina la institución "escogida" (Zapata etal., 2011). A nivel universitario, se produce un fenómeno de segmentación: el acceso a instituciones más o menos selectivas está determinado por el origen social del estudiante, denominado como "efecto cascada" por Brunner (2009).

En este contexto, los EPG han interesado recientemente a los investigadores chilenos en virtud de sus menores probabilidades de acceder a la universidad. El estudio de seguimiento de una cohorte de Castillo y Cabezas (2010) concluyó que la trayectoria escolar de los EPG presenta características particulares frente a sus pares que no ingresan a la universidad: (a) mejor rendimiento escolar; (b) realización de prácticas "estratégicas" para acceder a la universidad; (c) experiencia más adaptada a las normas escolares. No obstante, los EPG alcanzan menores resultados en la Prueba de Selección Universitaria (PSU) comparados con hijos de universitarios (Castillo y Cabezas, 2010; Centro De Políticas Comparadas de Educación [CPCE], 2009).

Canales y de los Ríos (2009) plantean que los EPG enfrentan problemas académicos como consecuencia de una enseñanza secundaria deficiente y falta de hábitos de estudio.. Por otro lado, la adaptación a la universidad es vivida como un "proceso estresante", debido a la socialización a nuevos códigos culturales, particularmente en universidades "de élite" donde son una minoría. De igual forma, Leyton etal. (2012)identifica un "sentimiento de desigualdad ante los compañeros" (p. 91) como una de las tensiones que ellos experimentan durante el ingreso a la universidad.

La prosecución de estudios de los EPG se vincula a su estatus socio-económico: dos de cada cinco EPG ingresan a la educación superior buscando mejorar sus condiciones económicas, mediante la obtención de mejores rentas que sus padres (CPCE, 2009). Una de sus principales motivaciones es conseguir seguridad económica (Canales y de los Ríos, 2009).

A pesar del creciente acceso de los EPG a las universidades, su permanencia es menor que los estudiantes no-EPG. Según Urzúa (2012) un 74\% de los jóvenes que abandonaron sus estudios era primera generación. La probabilidad de obtener un diploma es cercana al $70 \%$ para estudiantes con padres universitarios, en cambio para los EPG no sobrepasa el 38\%.

\section{Método}

Las preguntas de investigación que orientan este trabajo son: ¿Cómo los EPG construyen su experiencia universitaria? ¿Cuáles son los significados que se atribuyen a esta experiencia? Dado que la pregunta de investigación se encuentra dirigida a conocer el sentido subjetivo de las vivencias de los individuos, se escogió trabajar a 
partir del paradigma constructivista, puesto que su interés investigativo es la comprensión de los fenómenos; y que considera que las realidades sociales son múltiples y producidas por las personas, y en tanto, el conocimiento se construye en interacción entre el investigador y los participantes de la investigación (Guba y Lincoln, 2002). La metodología cualitativa, desde la cual se realizó el estudio, se caracteriza por la búsqueda de comprensión de "los acontecimientos, acciones, normas, valores, etc., desde la perspectiva de los propios sujetos que los producen y experimentan" (Vieytes, 2004, p. 613).

El método biográfico orientó el estudio en función de su preocupación de vincular a los sujetos con su contexto histórico y social, mediante el análisis de "experiencias personales que conectan entre sí "yos" individuales que interactúan en familias, grupos e instituciones" (Sautu, 1999, p. 21). Estos métodos pueden enfocarse en la vida completa de los individuos o en fragmentos significativos (Bertaux, 2010). Una de sus características principales es la relevancia dada al tiempo y la familia (Miller, 2000), este último aspecto especialmente relevante en la bibliografía revisada. De esta manera, la investigación se enfocó en el fragmento de tiempo que corresponde a la trayectoria universitaria, relevando el rol de la familia y los padres en la construcción de un proyecto de estudios universitarios.

La técnica de recolección de datos escogida fue la entrevista semiestructurada, puesto que constituyen un recurso privilegiado para acceder a la información desde la perspectiva del actor (...) permite obtener una información contextualizada y holística" (Vieytes, 2004, p. 661). Acerca de la muestra, su selección "no pretende representar una población con el objeto de generalizar, sino que se propone ampliar el abanico y rango de los datos" (Vieytes, 2004, p. 614).

Los criterios de selección de los entrevistados fueron, en primer lugar, la pertenencia a la categoría de estudiantes de primera generación. En segundo lugar, se optó por encuestar estudiantes de cursos más avanzados, tercer y cuarto año de universidad, esperando encontrar una capacidad reflexiva respecto de su propia experiencia, además de una trayectoria universitaria más larga. Las carreras escogidas fueron seleccionadas pensando en representar campos disciplinarios diferentes. Así se escogieron tres carreras: ingeniería comercial, enfermería y pedagogía en historia. Las universidades pertenecen a la ciudad de Concepción, Chile. Dos de ellas son denominadas universidades tradicionales y una corresponde a universidad privada. La decisión de seleccionar diferentes universidades y carreras se basó en el objetivo de encontrar diversidad en los relatos de los jóvenes.

El trabajo de campo finalizó con la realización de doce entrevistas a estudiantes de primera generación de tres universidades. Esta investigación se plantea como un estudio exploratorio, y debido a la pequeña muestra no pretende generalizar resultados, sino aportar nuevos elementos de corte cualitativo a la discusión sobre los estudiantes en educación universitaria.

El análisis de datos comenzó a partir de la búsqueda de conceptos comunes en cada una de las entrevistas y a partir de ellos se conformaron tres categorías y subcategorías de análisis. La primera categoría da cuenta de la construcción de un proyecto de 
estudios universitarios, con dos subcategorías que contribuyen a comprender este proceso, la influencia de los padres y la naturalización de estudiar en la universidad. La segunda categoría se denominó vida en la universidad, que incluye la subcategoría ser estudiante universitario y la integración. La tercera categoría aborda los significados que los estudiantes otorgan a su experiencia universitaria a través de tres subcategorías: movilidad social, vocación y la retribución a los padres.

\section{Principales resultados}

\section{El proyecto de estudiar en la universidad}

a) Influencia de los padres

Los relatos de la mayoría de los jóvenes revelan que la idea de estudiar en la universidad se origina en sus padres. Según estos estudiantes, el discurso de los padres estuvo siempre dirigido a promover el trabajo escolar: estudiar en la escuela y luego ingresar a la universidad. Los padres estaban fuertemente comprometidos con la educación de sus hijos, lo que se materializó en algunas decisiones estratégicas que favorecían el objetivo de acceder a la educación superior, como nos relata María ${ }^{3}$ (estudiante de enfermería):

"De hecho, mi mamá compró casa acá (Concepción), ahorraron harto tiempo, ahorraron mucho y como nosotros, parece que tenía sólo a mi hermana mayor ahorraban casi todo y juntaron plata y al final se compraron una casa acá, pensando en que nosotros pudiéramos estudiar acá. Como que siempre han hecho todo en pro de que nosotros estudiemos, de que tengamos educación superior".

A estas decisiones se agrega el trabajo de los padres, que, según relatan los entrevistados, es realizado muchas veces en condiciones precarias y mal pagados, todo aquello en función de entregar la mejor calidad de vida posible a sus hijos y, en particular, una buena educación. Por esto que los estudiantes se refieren a los esfuerzos y "sacrificios" de sus padres. El relato de Carolina (estudiante de ingeniería) revela esto.

"Mi papá trabaja para un vitivinícola en los viñedos, ahí trabaja metido en las viñas, haciendo esto, que podando, no sé mucho, pero eso, en el verano a todo sol, en el invierno, bueno en la lluvia los mandan pa' la casa, pero fríos en la mañana... Y mi mamá igual po', como te dije, si mi mamá no trabajara de temporera yo no podría estar estudiando acá, o sea, yo no podría estudiar".

Los relatos muestran que los padres son más que una influencia. Para los padres, la educación ocupa un "lugar simbólico" (Lahire, 1995): se comprometieron con los estudios de sus hijos. Los padres inscriben la educación de sus hijos en un "horizonte temporal" (Gofen, 2009), que implica la disposición a realizar esfuerzos considerables. Los entrevistados, influenciados por el aliento de los padres a estudiar en la

\footnotetext{
${ }^{3}$ Se utilizaron seudónimos para garantizar al anonimato de los entrevistados.
} 
universidad, constituyen un proyecto de futuro en este sentido. Por otro lado, el oficio de los padres juega un rol significativo en las expectativas de los jóvenes que aspiran a vivir en mejor condiciones que ellos.

b) Naturalización de estudiar en la universidad

Los discursos de los entrevistados revelan que la escolarización obligatoria, que en Chile es de 12 años, ha dejado de ser un referente de logro escolar. Asimismo, alcanzar este nivel educativo no permite acceder al mercado laboral en condiciones diferentes a las de sus padres. Para los entrevistados estudiar en la universidad es un evento que se concibe como parte de la vida. Se constituye, en efecto, en una opción naturalizada. María lo dice sin ambages: "Para mí no era un sueño ir a la universidad, sino que era como un hecho, yo tenía que estudiar en la universidad (...) claro, con becas, tenía que prepararme mucho". Los jóvenes no consideran que su entrada a la universidad sea extraordinaria, sino "totalmente natural" y "normal", que tampoco se concibe como una obligación. De hecho, sólo un estudiante reconoce que recibió "presión familiar" para estudiar en la universidad. Los padres tienen un rol significativo en esta concepción naturalizada de estudiar en la universidad, de acuerdo a lo analizado anteriormente.

\section{La vida en la universidad}

a) Ser estudiante universitario

Para los estudiantes entrevistados el primer año fue el más complejo debido a la adaptación a las mayores exigencias académicas comparadas a la secundaria. Este proceso implicó la búsqueda de un método eficiente para estudiar los contenidos y aprobar sus asignaturas. Es interesante notar que la forma de enfrentar los estudios difiere según la carrera.

Tanto los estudiantes de ingeniería como de enfermería han aprendido a adaptarse al ritmo académico de la universidad, de hecho, han aprobado casi la totalidad de los asignaturas, lo que, de acuerdo a sus relatos, es el resultado del desarrollo de hábitos de estudio y una adecuada gestión del tiempo. Las estudiantes de enfermería, adicionalmente, han debido desenvolverse como estudiantes en práctica en un hospital, y aparecen muy exigidas para obtener logros en sus asignaturas y prácticas.

El relato de Virginia (estudiante de enfermería) ilustra esto:

"No me costó adaptarme. Me costó el ramo no más, pero, adaptarme al ritmo universitario no. Lo que sí me ha costado con el tiempo, sacrificar mi tiempo personal, porque es una, puede que no sea una carrera tan dificil, bueno es dificil pero no es imposible, pero demanda demasiado tiempo. O sea, yo a veces me acuesto a la 3 de la mañana y me tengo que estar levantando a las 5 o a las 6 . Y no duermo en todo el día, ando todo el día en la U...". 
A diferencia de los estudiantes de ingeniería y enfermería, los estudiantes de historia han experimentado más dificultades para adecuarse a las exigencias universitarias. Algunos han superado lentamente sus problemas y se encuentran en un proceso de adquisición de hábitos de estudio. En cambio, otros estudiantes parecen poco comprometidos con su aprendizaje, declarando abiertamente que no tienen hábito de estudio. Este fenómeno se vincula con la categoría siguiente.

\section{b) La integración en la universidad}

La socialización en la vida universitaria ha implicado diversos desafíos para los estudiantes: exigencias académicas, nuevos espacio de estudio, compañeros/as, profesores/as, nuevas reglas y códigos culturales, que han sido vivenciados con diversos grados de dificultad, pero que no han involucrado sentimientos de no pertenencia o distancia cultural.

El énfasis que los entrevistados otorgan a las relaciones interpersonales revela que la universidad no es considerada únicamente como un lugar de adquisición de conocimientos, sino también un espacio que permite generar vínculos significativos. Las amistades representan para ellos un apoyo académico y personal importante. Por todo esto, la lógica de la integración es vivida intensamente por todos los entrevistados.

Ahora bien, cuando la lógica de la integración se asocia al aspecto más lúdico de la universidad, y el binomio relaciones interpersonales y diversión comienzan a tomar un rol demasiado preponderante, se producen consecuencias negativas en el rendimiento académico, como es el caso de los estudiantes de historia. Sus dificultades para dedicarse a los estudios se encuentran asociadas a las posibilidades lúdicas que ofrece el espacio universitario, donde las fiestas y sobre todo el alcohol juegan un papel importante. Daniela (estudiante de historia) profundiza al respecto:

“...pero me metí en grupos de amigos que claramente eran una mala influencia y no supe filtrar la amistad, sino que, no, y ahi me perdi un tiempo.

¿Pero ibas a clases?

No, o sea, yo llegaba a la U y ya con los compañeros, juntemos unas monedas y vamos a comprar, pasamos a comprar vino, fuera de la $U$ y tomando y fumando y ahi me sumergí. Fue un periodo de crisis feo, de hecho hasta los profesores se dieron cuenta y me decian "qué te pasa que no vienes a clases, te está yendo mal en las notas y tú puedes más" y ahi dije, ya "hay que empezar de nuevo".

En un balance más personal de este periodo, los estudiantes destacan que su ingreso a la universidad ha implicado un periodo de independencia y libertad, así como, la oportunidad de madurar, y aumentar tanto la autonomía respecto de los padres, como el sentido de la responsabilidad. En resumen: desarrollarse como persona. Los jóvenes que debieron emigrar desde un medio rural hacia la ciudad perciben positivamente el hecho de dejar la casa paterna, a pesar de las dificultades iniciales. 


\section{Significados atribuidos a la experiencia universitaria}

a) El significado de la movilidad social

Para estos jóvenes la búsqueda de la movilidad social es uno de los principales alicientes para realizar estudios universitarios. Por ejemplo, Jorge (estudiante de ingeniería) considera que lo más importante es "el título y obtener un profesión buena, para ganar buen sueldo, para asegurar el futuro, eso es lo primordial". De hecho, él encarna perfectamente el sentido utilitario. Andrea (estudiante de ingeniería) expresa su opinión de la manera siguiente:

"Mira yo sé que la plata no es el objetivo de vida, obvio que no, cachai, pero hay que decirlo, la plata te facilita mucho la vida, sin plata ahora tú no haces nada. Puedes ser muy buena persona, puedes tener muy buena gente alrededor tuyo pero sin plata no haces nada. Entonces en realidad hay que hacer equilibrio, tu familia va a ser feliz si tu familia no tiene necesidades, no pasa(...) no tiene problemas, cachai, y en eso te ayuda harto tener plata y por lo tanto tener un título, o sea, tener una buena pega, tener una seguridad, yo creo que eso más que nada".

La posibilidad de estudiar en la universidad, obtener un diploma, y luego un trabajo, es considerada como la promesa de encontrar la estabilidad y seguridad económica que sus padres no disponen. Son pocos los jóvenes que reconocen que sus familias son o eran pobres. En efecto, la obtención del título no se expresa, al menos no abiertamente, como una promesa de romper el círculo de la pobreza. La fórmula para expresar sus anhelos es la "estabilidad económica", aludiendo a la vulnerabilidad que han vivido.

El sentido de la movilidad social se manifiesta como un proyecto profesional bastante definido, que funciona como un incentivo para dedicarse a los estudios y obtener un diploma universitario. Sin embargo, la esperanza puesta en el título no es ingenua. Los jóvenes reconocen las dificultades del mercado de trabajo actual y consideran el título como una "base", un "apoyo" que "implica más facilidades" para insertarse en el mercado laboral, pero que no otorga una garantía, debido a la gran competencia por puestos de trabajo. Por lo anterior, los jóvenes han considerado algunas estrategias para facilitar su inserción. Una de ellas es seguir estudiando luego de obtener un primer diploma, predisponiéndose a una "lógica inflacionista". Estos elementos demuestran la importancia de la lógica estratégica en la experiencia universitaria.

b) El significado de la vocación

La primera significación de la experiencia universitaria de los jóvenes tiene relación con la vocación. El relato de Virginia ilustra este aspecto:

"Yo encuentro que mi mentalidad cambió totalmente desde el primer año de carrera, yo veía el mundo de otra forma, desde, lo veía como desde el enfoque de enfermería, o sea, si yo antes veía que las personas, como que te meten la enfermería desde el primer semestre encuentro yo porque yo veía, no sé po', que alguien no se 
cuidaba era como, ya un diabético que come torta, yo decía "ya que tan terrible va a ser que se coma una torta”, entonces ahora es como, es totalmente distinto para mi".

Las estudiantes de enfermería presentan un fuerte sentido de vocación, que se revela como una aspiración de ayudar y servir a otros, lo que motiva su trayectoria universitaria. Las "clínicas" no solo son un lugar de formación profesional, sino de transformación de la relación con los enfermos. La experiencia de compartir con enfermos graves impresiona fuertemente la sensibilidad de las jóvenes, quienes la conciben como una vivencia que las enriquece a nivel personal.

Para los estudiantes de pedagogía en historia, la vocación implica un compromiso con la formación de sus alumnos a través del desarrollo de su capacidad reflexiva y de una preocupación por el contexto social en el cual viven. Más aún, los estudiantes esperan un cambio de la sociedad a partir de las salas de clases. Su vocación se manifiesta en proyectos profesionales como enseñar en zonas rurales o en sectores urbanos marginales.

Los conocimientos adquiridos en la universidad son valorizados en función de un diploma que les abrirá las puertas del mercado de trabajo y los futuros beneficios que éste aportará en términos de movilidad social. No se considera al conocimiento por sí mismo. El sentido otorgado a los estudios puede ser abordado desde diferentes perspectivas. Una de ellas apunta a la relación con el saber, que es definido por como "relación de un sujeto con el mundo, con sí mismo y con los otros" (Charlot, 1997, p. 90). La investigación de Erlich y Boyer (2000) demuestra que los estudiantes de sectoresde menores ingresos, a diferencia de estudiantes de clase alta, establecen una relación con el saber más asociado al mundo laboral y profesional que a los saberes mismos. En este análisis, el diploma es central para conseguir un "buen empleo" y proyectar su bienestar económico. Por tanto, se establece una relación con el saber utilitarista. La preocupación por la empleabilidad, los ingresos y la movilidad conlleva una valorización de los conocimientos concretos y útiles (Lehmann, 2009).

En las entrevistas, la búsqueda de la utilidad no implica indiferencia a los saberes. Muchos jóvenes indican que están interesados en adquirir nuevos conocimientos, pero solamente un joven subraya el desarrollo intelectual gracias a la universidad. Esta es la principal diferencia respecto a la figura del heredero, que valoriza el conocimiento en sí mismo y no tanto por su rentabilidad futura. Para los entrevistados, la relación con el saber se establece a partir de la utilidad y, en este sentido, actúan plenamente en una lógica estratégica.

c) El significado de retribución a los padres

Un tercer significado otorgado por los entrevistados a estudiar en la universidad, se refiere a la oportunidad de retribuir los esfuerzos que sus padres han realizado para apoyarlos en su trayectoria escolar. Los estudiantes que manifiestan esta idea comparten dos características: son mujeres y proceden de localidades rurales. Carolina plantea la idea de la retribución directamente: 
"Yo entré a la universidad porque yo quiero ser una profesional y quiero devolver todo lo que ellos me dieron (...) yo quiero surgir".

En el relato de Yésica (estudiante de enfermería), se confunden el sentido de la movilidad social y el sentido de la retribución a los padres:

"Entonces a ellos les costó harto y el hecho de que ellos hayan sido pobres cuando chiquititos y después empezaron el matrimonio, después eran pobres, mi hermana, mis hermanas alcanzaron a vivir eso y ver que ahora les está yendo bien, que tienen su sueldito porque están trabajando, igual es súper gratificante para ellos. Y no, si están contentos, más que por el bien de ellos, porque ellos nunca nos han exigido nada, como que, ahora yo te pagué estos estudios, te ayudé a estudiar, ahora tienes que comprarme cosas o cosas así, no ellos nunca hacian eso, asi que están contentos por el bien que nos hicieron a nosotros. Pero ellas igual les dan sus regalitos, les compran cositas, les hacen pedidos, pero porque ellas quieren hacerlo".

Para las entrevistadas el sentido de la movilidad social se vincula al sentido de la retribución cuyo propósito es recompensar los esfuerzos de sus padres. En efecto, un diploma universitario representa, para ellas y sus familias, la promesa de mejores condiciones de vida que podrá concretarse gracias a la obtención de un título profesional y un trabajo bien remunerado. Por otro lado, las estudiantes manifiestan una aspiración de independencia económica respecto a un futuro marido. Así el título profesional viene a representar también un símbolo de autonomía femenina.

Es bastante interesante que el sentido de la retribución solo haya sido manifestado por mujeres. ¿Qué puede significar esto? Una explicación posible remite al concepto de "estereotipos sexuales" en los cuales "cada niño y cada niña está obligado a construir su identidad personal tomando posición en relación a las expectativas sociales tradicionalmente propias a su sexo" (Baudelot y Establet, 1992, p. 72). Según los estereotipos sexuales dominantes, los roles de hombres y mujeres son diferenciados. Los hombres son los proveedores del hogar y las mujeres están encargadas del bienestar familiar. Estas representaciones de lo femenino y masculino parecen estar muy presentes en la sociedad chilena. ¿Qué sucede en el marco de una economía familiar frágil? Una hipótesis posible es que las estudiantes de primera generación planean asumir responsabilidades respecto a la economía familiar. Así, para ellas, su rol de apoyo familiar no cambia, sino que se agrega un segundo, proveedora. El sentido de la retribución parece desarrollarse en tanto que responsabilidad familiar y se expresa como una suerte de "solidaridad familiar". De este modo, los padres no son los únicos involucrados sino todos los miembros de la familia. En este aspecto es importante decir que si bien las estudiantes subrayan que sus padres no exigen ni esperan una recompensa por su preocupación y trabajo: estudiar en la universidad se conforma como un proyecto familiar de movilidad social.

\section{Discusión y conclusiones}

Los EPG entrevistados presentan una experiencia universitaria a partir de la cual articulan las lógicas de acción de la experiencia. La lógica estratégica se expresa a 
través de la definición de un proyecto profesional, que apunta a la inserción en el mercado laboral. La fuerte influencia de la lógica estratégica en los estudiantes no impide que la dimensión de la integración y la vocación adquieran un papel significativo para los jóvenes. A pesar de los desafíos que plantea articular las lógicas de acción, los entrevistados logran aliviar las tensiones, y, además, otorgar sentido a sus estudios, combinando los aspectos de la experiencia universitaria en su manifestación "fuerte". Ningún estudiante se encuentra cercano a la figura del malheur etudiant, ya que todos manifiestan, un "proyecto profesional" e integración al medio universitario.

La experiencia universitaria de los entrevistados se comprende a partir de tres significaciones: movilidad social, vocación y retribución a los padres. Estos tres sentidos se definen en relación al diploma, en otras palabras, toda la experiencia universitaria se orientan hacia su obtención. Sin embargo, esto no implica que la utilidad sea la única significación, dado que en la experiencia universitaria de la mitad de nuestros entrevistados, la lógica estratégica y la vocación funcionan en conjunto. La lógica vocacional actúa como un motor para comprometerse con sus estudios, permitiéndoles construir un "proyecto profesional". Pero aquí la vocación se desarrolla en un sentido diferente al propuesto por Dubet (1994a). Ella no es vivida como una realización intelectual gracias a la adquisición de nuevos conocimientos. Para estos jóvenes, la vocación, influenciada también por el tipo de muestra escogida, se desarrolla como una relación con otras personas. El conocimiento es valioso en la medida que permitirá trabajar ayudando a otros. Siguiendo a Felouzis (2001), los estudiantes necesitan definir sus objetivos y encontrar una significación personal a sus estudios universitarios para poder comprometerse efectivamente con una carrera.

Una de las principales conclusiones de esta investigación es la importante influencia de los padres en la trayectoria escolar de los EPG entrevistados. A menudo, se dice que estos estudiantes llegaron a la universidad "a pesar" de su origen familiar. Por el contrario, de acuerdo a los relatos de los entrevistados, sus padres fueron la principal fuente de apoyo para un proyecto de estudios universitarios, a través de un discurso y prácticas que enfatizaba la importancia de estudiar. Para ellos, ir a la universidad devino en una opción naturalizada: ser estudiante universitario es una parte de la vida. Cabe preguntarse en qué condiciones sociales y familiares los estudiantes construyen un proyecto movilidad social, que pueda actuar como un facilitador la integración en la universidad, siguiendo los planteamientos Gofen (2009), y Galland y Oberti (1996).

Las principales tensiones son experimentadas durante el primer año de universidad, las que, sin embargo, son matizadas por los estudiantes debido a logros como la aprobación de ramos y la adopción de un ritmo de estudio apropiado a las exigencias universitarias. No obstante, algunos de los estudiantes entrevistados presentan dificultades para desarrollar hábitos de estudios y, en definitiva, fortalecer su compromiso con su carrera. Esto da cuenta de problemas académicos que perduran incluso luego de varios años de haber egresado de la enseñanza media, revelando carencias que pueden relacionarse con el capital cultural del estudiantes. En este sentido, nuevas interrogantes surgen respecto al rol de las universidades en la 
implementación de políticas compensatorias para jóvenes provenientes de entornos desfavorecidos.

A pesar de las tensiones mencionadas, no se identifica un discurso en torno a dificultades de socialización en la universidad ni de "distancia cultural". Los vínculos entre pares son buscados y apreciados. En efecto, los entrevistados no experimentaron grandes dificultades en la integración en la universidad, ni relatan haberse sentido en un "mundo ajeno". Esto constituye una diferencia respecto a otras investigaciones, que aludían a la lejanía a nivel cultural y de códigos implícitos incomprendidos. En este sentido, cabe preguntarse cuál es el impacto que puede tener el origen social de los estudiantes que acoge una universidad en las posibilidades de integración, tanto académica como social, de los EPG.

Los resultados de esta investigación deben ser analizados considerando su carácter exploratorio. A pesar de esto, la perspectiva de la experiencia universitaria puede constituirse en un ámbito de investigación que aporte nuevos elementos de análisis en un sistema de educación superior desigual y segmentado, que afecta especialmente a los jóvenes provenientes de los sectores más desfavorecidos, como los EPG.

\section{Referencias bibliográficas}

AUClAIR, R., BELANGER, P., DORAY, P., GALLIEN, M., GROLEAU A., MASON, L., MERCIER, P. (2008). Les étudiants de première génération: un concept prometteur? Transitions - Note 2. Montréal: Fondation canadienne des bourses d'études du millénaire.

BAUDELOT, C., ESTABLET, R. (1992) Allez les filles. Paris: Editions du Seuil.

BEAUD, S. (2002).80\% au bac...et après. Les enfants de la démocratisation scolaire. Paris: La Découverte.

BERTAUX, D. (2010). Le récit de vie. Paris: Arman Colin.

BOYER, R., CORIDIAN, C., ERLICH, V. (2001). L'entrée dans la vie étudiante. Socialisation et apprentissages. Revue française de pédagogie, 136, 97-105.

BOURDIEU, P., PASSERON, J.C. (1964). Les héritiers. Les étudiants et la culture. Paris: Les éditions de minuit.

BRUNNER, J.J. (2011). Educación Superior en Ibero América: Informe 2011.Santiago de Chile: Centro Interuniversitario de Desarrollo.

BRUNNER, J.J. (2009) Educación superior en Chile. Instituciones, mercados y políticas gubernamentales (1967-2007). Santiago de Chile: Ediciones Diego Portales.

CANALES, A., DE LOS RÍOS, D. (2009). Retención de estudiantes universitarios vulnerables. Calidad en la Educación, 30, 50-83.

CASTILLO, J., CABEZAS, G. (2010). Caracterización de jóvenes primera generación en educación superior. Nuevas trayectorias hacia la equidad educativa. Calidad de la Educación, 32, 44-76. 
CENTRO DE INVESTIGACIÓN Y DESARROLLO DE LA EDUCACIÓN, CIDE. (2012). IX Encuesta Actores del Sistema Educativo. CIDE-Universidad Alberto Hurtado. Recuperado de http://www.cide.cl/encuestas.php

CENTRO DE POLÍTICAS COMPARADAS DE EDUCACIÓN CPCE (2009). Primera generación en la universidad. Informe Preliminar. Universidad Diego Portales.

CHARLOT, B. (1997). Du rapport au savoir. Eléments pour une théorie. Paris: Anthropos.

CHARLOT, B. (1999). Le rapport au savoir en milieu populaire. Une recherche dans les lycées professionnels de banlieue. Paris: Anthropos.

CHEN, X., CARROLL, C. (2005) First-Generation Students in Postsecondary Education A Look at Their College Transcripts Postsecondary Education. Descriptive Analysis Report. Project Officer. National Center for Education Statistics. United States.

DUBET, F., MARTUCCELLI, D. (1996). A l'école. Sociologie de l'expérience scolaire. Paris: Seuil.

DUBET, F. (1994a). Sociologie de l'expérience. Paris: Seuil.

DUBET, F. (1994b) Dimensions et figures de l'expérience étudiante dans l'université de masse. Revue française de sociologie, 35 (4), 511-532.

ERLICH, V., BOYER, R. (2000). La socialisation des étudiants débutants. Expériences universitaires, familiales et sociales. Les dossiers, 115. Ministère de l'éducation national, France.

ESPINOZA, O., GONZÁLEZ, L., LATORRE, C. (2009). Un modelo de equidad para la educación superior: Análisis de su aplicación al caso chileno. Revista de la Educación Superior, 38 (35), 97-112.

FELOUZIS, G. (2008). Des mondes incertains: les universités, les diplômés et l'emploi. Formation emploi, 101, 135-147.

FELOUZIS, G. (2001). La condition étudiante. Sociologie des étudiants et de l'Université. Paris: PUF.

GALLAND, O., OBERTI, M. (1996). Les étudiants. Paris: La Découverte.

GOFEN, A. (2009). Family Capital: How first-generation higher education students break the intergenerational cycle. Family Relations, 58, 104-120.

GROLEAU, A., DORAY, P., KAMANZI, C., MASON, L. (2010). Les possibilités et les limites d'une importation conceptuelle. Les étudiants de première génération au Canada. Education et sociétés, 26, 107-122.

GUBA, E. Y LINCOLN, I. (2002). Paradigmas en competencias en la investigación cualitativa. En DENMAN, C. Y HARO, J.A. (comps.) Por los rincones. Antología 
de métodos cualitativos en la investigación social (pp. 113-145). Sonora: El Colegio de Sonora.

LAHIRE, B. (1995). Tableau de famille. Heurs et malheurs scolaire en milieux populaires. Paris: Le Seuil.

LEHMANN, W. (2012) Working-class students, habitus, and the development of student roles: a Canadian case study. British Journal of Sociology of Education, 33 (4), 527-546.

LEHMANN, W. (2009) University as vocational education: working-class students' expectations for university. British Journal of Sociology of Education, 30 (2), 137149.

LEYTON, D. VASQUEZ, A., FUENZALIDA, V. (2012). La experiencia de estudiantes de contextos vulnerables en diferentes instituciones de educación universitaria (IESU): resultados de investigación. Calidad en la Educación. 37, 61-97.

MILLER, R. (2000). Researching Life Stories and Family Histories. Londres: Sage.

REAY, D., CROZIER, G. \& CLAYTON, J. (2010). 'Fitting in' or 'standing out': working class students in UK higher education. British Educational Research Journal, 36 (1), 107-124.

ROLANDO, R., SALAMANCA, J., ALIAGA, M. (2010). Evolución Matrícula Educación Superior de Chile, periodo 1990-2009. Sistema Nacional de Información de la Educación Superior (SIES) de la División de Educación Superior. Santiago: MINEDUC.

SAUTU, R. (1999). El método biográfico: la reconstrucción de la sociedad a partir del testimonio de los actores. Buenos Aires: Editorial Belgrano.

URZÚA, S., (2012). Rentabilidad de la educación superior en Chile. Revisión de las bases de 30 años de políticas públicas. Estudios Públicos, 125, 1-52.

VIEYTES, V. (2004). Metodología de investigación en organizaciones, mercado y sociedad: epistemología y técnicas. Buenos Aires: Editorial de las Ciencias.

ZAPATA, G. (COORD.); TEJEDA, I., ROJAS, Á. (2011). Educación Superior en Chile-Informe Nacional. En: BRUNNER, J.J. (2011). Educación Superior en Ibero América: Informe 2011. Santiago de Chile: Centro Interuniversitario de Desarrollo.

\section{Correspondencia con los autores}

Valentina Javiera SOTO HERNÁNDEZ

Dirección de Docencia - Universidad de Concepción

Edmundo Larenas 64-A

4030000 Concepción (Chile)

e-mail: valentinasoto@udec.cl 